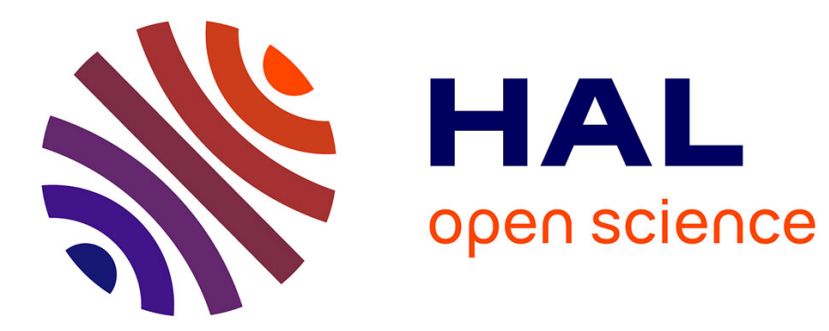

\title{
Les seniors au festival
}

Aurélien Djakouane, Marie-Thérèse Jourda, Emmanuel Négrier, Marion Vidal

\section{To cite this version:}

Aurélien Djakouane, Marie-Thérèse Jourda, Emmanuel Négrier, Marion Vidal. Les seniors au festival.

Gérontologie et Société, 2011, 3 (138), pp.195 - 216. 10.3917/gs.138.0195 . hal-01436577

\section{HAL Id: hal-01436577 https://hal.science/hal-01436577}

Submitted on 16 Jan 2017

HAL is a multi-disciplinary open access archive for the deposit and dissemination of scientific research documents, whether they are published or not. The documents may come from teaching and research institutions in France or abroad, or from public or private research centers.
L'archive ouverte pluridisciplinaire HAL, est destinée au dépôt et à la diffusion de documents scientifiques de niveau recherche, publiés ou non, émanant des établissements d'enseignement et de recherche français ou étrangers, des laboratoires publics ou privés. 


\title{
Les seniors au festival :
}

\section{Le critère de l'âge dans l'analyse des pratiques et des goûts culturels}

\author{
Aurélien Djakouane, \\ Centre Norbert Elias (UMR 8562) et CEPEL (UMR 5112), aurelien.djakouane@voila.fr \\ Marie Jourda, \\ CEPEL, Université Montpellier 1, marie.jourda@univ-montp1.fr
}

Emmanuel Négrier, CEPEL, Université Montpellier 1, negrier@univ-montp1.fr

Marion Vidal LARA/ICD Paris et LAMETA, Université Montpellier 1, mvidal@groupe-igs.fr

\section{RÉSUMÉ}

L'objectif de cet article est d'éclairer les goûts musicaux et les pratiques culturelles d'une certaine catégorie de personnes âgées : les festivaliers. En partant d'une enquête réalisée en 2008 auprès d'une cinquantaine de festivals de musique et de danse, nous cherchons à approfondir la réflexion sur la variable de l'âge dans l'analyse des pratiques festivalières et des goûts musicaux, à partir du cas des plus de 65 ans qui représentent $20 \%$ des festivaliers.

Nous montrons qu'il n'existe pas de festivals de "seniors », tout comme il n'existe pas une palette de goûts musicaux propre à chaque génération. Cependant, celle des festivaliers âgés se distingue de celle des jeunes. Ces différences de goûts et de pratiques semblent être croissantes avec l'écart entre les populations. Plus les écarts entre les tranches d'âge s'élèvent et plus les différences de goût s'accroissent.

\begin{abstract}
The aim of this paper is to shed light on musical tastes and cultural practices of a certain category of older people: the festival. Starting from a survey we conducted in 2008 with a fifty festivals of music and dance, we seek to deepen the reflection on the age variable in the analysis of practices and musical tastes, from the case of more than 65 years that represent $20 \%$ of festival goers.

We show that there are no festivals "seniors". Each generation haven't a palette of tastes However, old festival-goers have a range of cultural practices distinct from that of youth. These differences in tastes and practices appear to be increasing with the difference between populations. More differences between age groups and more the amount of taste differences are increasing.
\end{abstract}


Publié en 1993, l'ouvrage de Paul Paillat constitue le seul panorama disponible sur les pratiques culturelles des personnes âgées. Son travail s'appui essentiellement sur les trois éditions disponibles à l'époque de l'enquête sur les pratiques culturelles des Français du ministère de la Culture, celles de 1973, de 1981 et de 1989. Si ce travail n'a pas encore fait l'objet d'une réactualisation, il comporte néanmoins quelques données de cadrage intéressantes. D'emblée, l'avant-propos attire notre attention sur le fait qu'il existe des attitudes communes aux «anciens » qui les différencient du reste de la population, et qui justifient une approche générationnelle des pratiques culturelles ${ }^{1}$. Entre 1993 et 2009, le nombre des 60 ans et plus est passé de 11 à 14 millions de personnes, pour représenter aujourd'hui $23 \%$ de la population nationale. Si cette tendance semble se prolonger dans les années à venir ${ }^{2}$, les personnes âgées de demain ayant bénéficié d'une scolarité plus longue et de plus de loisirs, il est probable que leurs pratiques culturelles s'en trouvent modifiées.

En outre, Paillat insiste sur l'importance de scinder la catégorie des «plus de 60 ans » en deux, notamment en raison des changements importants qui adviennent avec « le quatrième âge ». Les clivages générationnels qu'il souligne rappellent combien les seniors d'aujourd'hui restent attachés à la culture de l'écrit dans laquelle ils sont nés, et dans laquelle ils ont forgé leurs pratiques et leurs goûts. La comparaison entre les années permet de pointer l'essor que les pratiques de sorties ont connu auprès de cette population. Cet essor s'explique à la fois par un accroissement et une diversification du volume de l'offre culturelle mais aussi par une amélioration constante des conditions de vie et de vieillissement. En 2008, la propension à sortir le soir a sensiblement augmenté chez les plus de 45 ans, et doublé chez les 65 ans et plus, notamment en ce qui concerne les sorties au cinéma et dans les salles de spectacles (Donnat, 2009). Malgré ces évolutions, les inégalités sociales d'accès à la culture demeurent particulièrement saillantes au sein d'une population qui n'a pas connu la massification de l'enseignement. Toutefois, si la diversification de l'offre et des supports culturels entraîne une légère diminution de la fréquentation régulière ou assidue des plus jeunes, c'est le contraire qui s'observe chez les plus de 55 ans. Ainsi, la dernière enquête sur les pratiques culturelles des Français constate un phénomène de vieillissement du public des habitués des équipements culturels qui suit l'allongement de l'espérance de vie : "si les 55 ans et plus représentent désormais $23 \%$ des personnes ayant une fréquentation régulière ou habituelle, contre 15\% onze ans plus tôt, c'est en raison de leur poids constant dans la société française mais aussi d'une atténuation des effets du vieillissement sur les sorties à l'âge de la retraite » (Donnat, 2009 , p. 196). Malgré ces évolutions, sur le plan global, le fait d'être âgé reste un handicap majeur dans l'accès à la culture. En 2008, 45\% des 65 ans et plus n'ont pas fréquenté une seule fois un équipement culturel au cours des douze derniers mois. Pour autant, cette même année, l'enquête que nous avons réalisée auprès d'une cinquantaine de festivals de musique et de danse met en évidence l'importance des plus de 65 ans qui représentent environ $20 \%$ des publics étudiés (Djakouane, Jourda et Negrier 2010).

En prenant appui sur cette étude, l'objectif de cet article est d'approfondir, à partir du cas des plus de 65 ans, la réflexion sur la variable de l'âge dans l'analyse des pratiques festivalières et des goûts musicaux. Y'a-t-il une spécificité des goûts et des pratiques des seniors par rapport aux autres festivaliers. Observe-t-on une hétérogénéité des comportements ? Les personnes âgées ont-elles des goûts moins éclectiques?

\footnotetext{
${ }^{1}$ A ce propos, cf. « Approche générationnelle des pratiques culturelles et médiatiques », in Culture prospective, $\mathrm{n}^{\circ} 3,2007$.

${ }^{2}$ Les projections de l'INSEE estiment que les 60 ans et plus représenteront $29 \%$ de la population en 2030 et $32 \%$ en 2050.
} 
Pour répondre à ces questions, nous allons mobiliser les résultats d'une enquête quantitative menée auprès des publics de 49 festivals de musique et de danse. Réalisée en 2008, cette étude a porté sur un panel de 207 spectacles réunissant des esthétiques variées allant des musiques classiques (50\% des spectacles), aux musiques actuelles et du monde $(15 \%)$, en passant par le jazz, la chanson et les variétés $(10 \%)$, la musique contemporaine $(12 \%)$ et la danse contemporaine (10\%). Nous avons également recherché à restituer la diversité géographique de l'offre festivalière (18 régions françaises sont représentées) et son étalement sur l'année, de l'avant-saison à l'après-saison en passant par le cœur de la saison estivale. Au total, 23344 questionnaires ont été recueillis et traités. Chaque questionnaire se composait de 23 questions réunies en quatre grandes thématiques : les modalités de la fréquentation des festivals (motivations, budget, ancienneté, accompagnement, nombre de spectacle...); les pratiques culturelles par ailleurs; les goûts musicaux et chorégraphiques; le profil sociodémographique des festivaliers (âge, sexe, professions et catégories socioprofessionnelles, niveau d'étude, lieu d'habitation...).

Toutes ces données, nous permettront d'aborder la question des pratiques festivalières des seniors en trois temps. Nous étudierons tout d'abord la typologie des festivals qui attirent plus particulièrement les plus de 65 ans et le mode de fréquentation des festivals. Ensuite, nous mettrons en parallèle, les goûts des festivaliers de plus de 65 ans avec ceux des moins de 40 ans afin de pointer l'hétérogénéité des goûts qui caractérise chaque classe d'âge. Enfin, nous discuterons de l'impact de l'âge relatif sur la participation culturelle dans le but de savoir si cette variable continue d'être un descripteur pertinent des comportements culturels.

\section{LES SENIORS DANS LES FESTIVALS}

Dans quelle proportion les festivals de musique attirent-ils les personnes âgées ? Ces dernières ont-elles un genre de musique de prédilection? Sont-elles nombreuses à se déplacer pour assister à un ou plusieurs spectacles ?

Cette première partie traite de l'ancrage générationnel des pratiques d'écoute musicale et de la manière dont l'offre festivalière restitue ce phénomène. Pour cela, nous partirons des données nationales sur les pratiques d'écoute musicale des différentes classes d'âge de la population, pour discuter ensuite de la place que les seniors occupent parmi les publics des festivals et de la manière dont ils s'orientent dans l'offre festivalière.

\subsection{L'écoute musicale : un phénomène générationnel ?}

En 1997, les 65 ans et plus étaient seulement 4\% à écouter de la musique tous les jours ou presque, ils sont $10 \%$ en 2008 . Cette importante progression s'inscrit dans un mouvement général qui touche l'ensemble des classes d'âge et qui s'explique, notamment, par l'évolution des supports techniques, la baisse du coût des équipements, et l'accroissement constant de l'offre musicale. Comme le rappelle Olivier Donnat, "la musique semble bénéficier d'une dynamique générationnelle qui ne se dément depuis les années $1970 »$ (p. 121) qui consacre un «boom musical» qui n'a toujours pas cessé. Toutefois, et même si le renouvellement générationnel fait que les adultes sont plus nombreux qu'avant à écouter de la musique régulièrement, l'écoute musicale quotidienne reste un phénomène caractéristique de la jeunesse. En effet, rares sont aujourd'hui ceux parmi les moins de 45 ans qui n'écoutent pas de musique : "pour la majorité d'entre [eux], il s'agit d'une activité totalement intégrée ans le quotidien qui accompagne une grande partie de leurs activités » (p. 128). Pour donner des chiffres, $74 \%$ des $15-19$ ans écoutent de la musique tous les jours, ils ne sont que $10 \%$ chez les 65 ans et plus ; 5\% des 15-19 ans n'écoutent jamais de musique, ils sont $72 \%$ chez les 65 
ans et plus. Ces écarts générationnels se doublent d'une fracture sociale, certes moins soutenue, mais bien présente : $31 \%$ des ouvriers écoutent de la musique tous les jours ou presque, $41 \%$ des cadres et professions intellectuelles supérieures; $42 \%$ des ouvriers n'écoutent jamais de musique contre $26 \%$ chez les cadres.

D'une autre manière, l'observation des préférences musicales de la population française obéit à ce même clivage. A l'échelle nationale, les préférences se répartissent comme suit : 33\% préfèrent les variétés et la chanson françaises, $10 \%$ le rock et la pop, $9 \%$ la variété internationales et le R'n'B, et 9\% la musique classique et l'opéra. Rap et hip-hop, musiques du monde et traditionnelle, techno et musiques électroniques comptent respectivement pour $4 \%$, le jazz pour 3\%. L'éclatement des préférences musicales semble désormais s'installer comme une caractéristique structurelle de l'écoute musicale. Au-delà de la domination de la variété et de la chanson françaises, les catégories musiques du monde et musiques traditionnelles apparaissent, telles que le rock et la musique classique, comme des genres relativement consensuels, "à la fois de personnes aux profils différents et rejeté par une très faible proportion de Français (5\%)» (p.133). A l'opposée, le rap, le hip-hop et les musiques électroniques sont celles qui fédèrent le plus de rejet, respectivement $44 \%$ et $36 \%$ des Français n'écoutent jamais ou n'aiment pas ces registres. De fait, les genres musicaux participent d'un jeu complexe de différenciation qui renvoie essentiellement à des questions d'âge et de génération. Au-delà du clivage jeunes/âgés, l'analyse des préférences musicales générationnelles accentue un autre clivage, celui des musiques savantes et des musiques populaires, particulièrement saillants chez les plus de 65 ans. En cela, la logique générationnelle n'annule pas les effets liés au capital culturel ou à la position sociale. A l'échelle nationale, les classes supérieures manifestent une légère réserve à l'égard des variétés ainsi qu'une hostilité plus forte envers le hip-hop et le rap tandis qu'elles privilégient la musique classique, le jazz, l'opéra et, fait nouveau qui traduit l'avancée des générations, le pop-rock. A ce titre, Olivier Donnat rappelle qu'aujourd'hui, « la musique pop et rock atteint un niveau de diffusion dans les milieux favorisés comparable à celui de la musique classique et les écarts entre cadres supérieures et ouvriers sont à peu près du même ordre sur ces deux formes musicales » (p. 136), si bien qu'on peut se demander s'il faut continuer de parler de musique populaire à propos du rock. Le croisement des classes sociales et des générations crédite cette hypothèse. L'unité prétendue de l'univers musical des jeunes est toute relative, et varie largement suivant le sexe et l'origine sociale : $35 \%$ des moins de 30 ans issus des classes supérieures déclarent le rock comme genre musical préféré contre $11 \%$ de leurs homologues des classes populaires qui, pour leur part, citent plutôt les variétés et le R'n'B (24\%) ou le rap et le hip-hop (17\%). Cette progression de la musique rock se prolonge dans les tranches d'âges supérieures mais cesse au-delà d'un certain âge. Le jazz et la musique classique sont les deux autres genres musicaux qui obéissent à ce double ancrage social et générationnel. A toute conclusion hâtive qui conduirait à présenter de manière homogène l'univers musical des différentes générations, doit correspondre une étude plus nuancée des préférences à la lumière des clivages culturels et sociaux qui demeurent à l'intérieur du mouvement permanent qui caractérise l'évolution des répertoires musicaux et de leur diffusion.

\subsection{Les festivals des seniors}

Parmi les 49 festivals de musique et de danse que nous avons étudiés en 2008, les plus de 65 ans représentent près de $20 \%$ d'un public dont l'âge moyen se situe autour de 51 ans. Toutefois, ces seniors ne se répartissent pas de manière homogène parmi les différentes esthétiques que nous avons étudiées. La figure 1 nous permet de pointer certains cas extrêmes, comme le festival d'Antibes (dont l'âge moyen des festivaliers est de 63 ans) et les festivals 
de Saintes, de Saô̂ chante Mozart ou du Vigan, dont l'âge moyen est de 60 ans. En accord avec les données nationales, ces situations extrêmes se réfèrent à une programmation de musiques savantes (baroque, classique, contemporain) où les plus de 65 ans sont massivement présents. En revanche, ils sont nettement moins représentés parmi les publics des festivals de musiques actuelles et de musiques du monde.

Figure 1. L'âge moyen des publics suivant les festivals

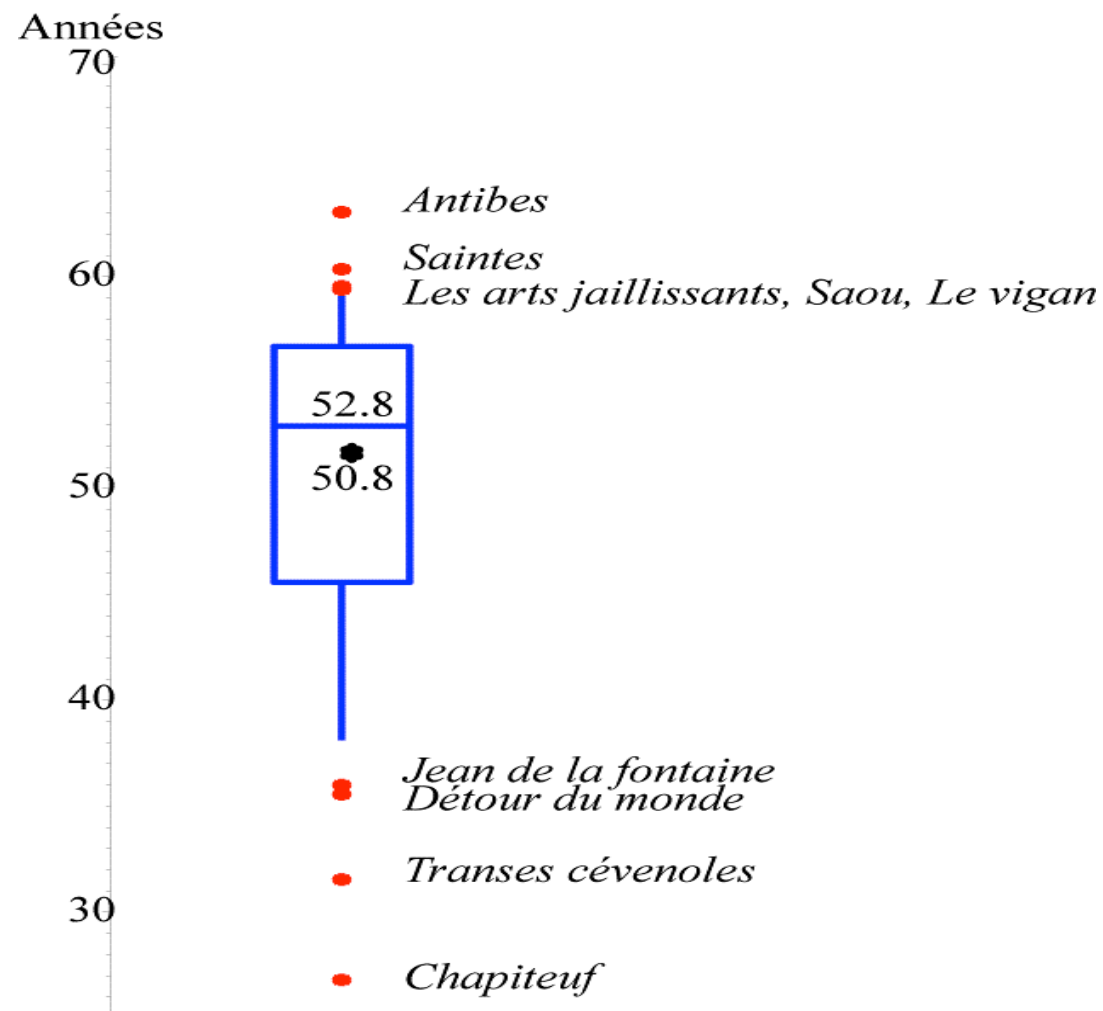

Légende: Pour comprendre ce graphique (un boxplot), il faut considérer que la moitié des festivals est comprise dans le rectangle. Le point indique la moyenne et la barre qui sépare le rectangle en deux parties correspond à la médiane. Celle-ci sépare les festivals en deux parties égales. Ceci veut dire, par exemple, qu'il y a autant de festivals dont la moyenne d'âge du public se situe sous 52,8 ans que de festivals qui dépassent cet âge.

Source : Enquête sur les publics des festivals de musique et de danse 2008, ( $\mathrm{n}=23$ 344).

Quel type de programmation attire les seniors? Les retrouve-t-on de façon homogène quelque soit le type de musique programmée ou sont-ils au contraire concentrés sur certains genres ?

Tableau 1. Les classes d'âge suivant le genre de spectacle (en\%).

\begin{tabular}{|lrrrrr|} 
Spectacle & Moins de 35 ans & $35-50$ ans & 50-65 ans & Plus de 65 ans & Total \\
\hline Baroque & 13,1 & 15,8 & 43,2 & 27,9 & 100,0 \\
\hline Classique & 17,4 & 13,9 & 39,2 & 29,5 & 100,0 \\
\hline Contemporain & 20,1 & 16,0 & 38,4 & 25,5 & 100,0 \\
\hline Jazz & 24,0 & 25,3 & 40,3 & 10,4 & 100,0 \\
\hline Chanson & 25,0 & 22,5 & 42,9 & 9,6 & 100,0 \\
\hline Danse & 34,2 & 23,6 & 30,4 & 9,8 & 100,0 \\
\hline Monde & 39,5 & 24,4 & 27,4 & 8,7 & 100,0 \\
\hline Actuelles & 67,4 & 22,9 & 8,6 & 1,1 & 100,0 \\
\hline Moyenne & 26,1 & 18,9 & 35,7 & 19,3 & 100,0 \\
\hline
\end{tabular}


Source : Enquête sur les publics des festivals de musique et de danse 2008, (n = 23 344).

Alors que les moins de 35 ans représentent $67,4 \%$ du public des festivals de musique actuelle, les seniors n'y sont presque pas présents $(1,1 \%)$. Les plus de 65 ans se retrouvent principalement dans les festivals de musique savante (baroque, classique et contemporain). On peut y voir ici un choc des générations avec deux extrêmes : les festivals de musiques savantes ont un public plutôt âgé alors que les festivals de musiques actuelles plutôt jeune. Cependant, on note que les plus jeunes sont "plus ouverts » que leurs aînés puisque leur présence représente environ $15 \%$ du public des musiques savantes alors que les plus de 65 ans comptent pour seulement $10 \%$ des festivals de musique actuelles (jazz, chanson, monde et actuelles confondus).

$* * *$

Les seniors ne se répartissent donc pas de manière homogène parmi les différentes esthétiques musicales, faisant la part belle aux musiques savantes. De fait, y compris à l'échelle des pratiques festivalières, l'ancrage générationnel des pratiques d'écoute musicale semble un phénomène qu'il faut lire en parallèle de la montée de l'éclectisme des goûts musicaux. Pour autant, ce constat ne doit pas faire oublier que de génération en génération les répertoires de goûts évoluent et que si les clivages demeurent entre les jeunes et les moins jeunes, ils ne portent pas sur les mêmes goûts musicaux.

Peu surprenant, ce clivage générationnel n'est pourtant pas le seul enseignement du travail que nous avons mené sur les festivals. En effet, la question que nous souhaitons aborder dans la partie qui suit propose d'approfondir la réflexion sur la variable de l'âge dans l'analyse des pratiques festivalières en s'interrogeant notamment sur l'éclectisme des pratiques culturelles et des goûts des ainés. Il s'agira ici d'interroger la pertinence de cette catégorie des «plus de 65 ans » qui revient de manière récurrente dans le découpage générationnel des pratiques culturelles. Cette catégorie est-elle réellement opératoire? Traduit-elle une réalité générationnelle incarnée par un corps de valeurs et de pratiques ? Ou bien, au contraire, s'agit-il d'un regroupement dont la commodité statistique n'épuise pas la réalité des pratiques d'une population hétérogène et relativement méconnue ?

\section{LES SENIORS : UNE CATEGORIE ET DES GOUTS HETEROGENES}

L'évolution des politiques publiques en faveur des personnes âgées a favorisé l'émergence d'une catégorie nouvelle, celle des seniors. Pour autant, ce terme n'en recouvre pas une réalité hétérogène et difficile à cerner. En restant concentré sur nos festivaliers, nous tenterons de savoir qui sont les seniors des festivals, et notamment quels sont leurs goûts musicaux ? Mais avant de répondre à cette question, nous tenterons d'y voir plus clair dans les différentes catégories qui permettent des désigner les personnes âgées aujourd'hui. Ce détour nous permettra d'aller plus en avant dans la description de la population festivalière étudiée.

\section{1. «Senior, plus de 65 ans, $3^{\text {ème }}, 4^{\text {ème }}$ âge... » : comment s'y retrouver ?}

Avec le vieillissement de la population qui s'impose comme une caractéristique structurelle forte de l'évolution des sociétés occidentales contemporaines, de nouveaux termes sont apparus pour désigner les fractions les plus âgées de la population. En raison de l'augmentation de l'espérance de vie, l'âge de la vieillesse apparaît désormais comme une borne extrêmement variable et symbolique qui explique en partie l'apparition d'une catégorie telle que celle du $4^{\text {ème }}$ âge. 
En 1962, le rapport Laroque qui prônait une politique d'insertion sociale des personnes âgées, a contribué à l'émergence d'un $3^{\text {ème }}$ âge composé de personnes bien intégrées dans le circuit de consommation ${ }^{3}$. Cette catégorie fera naître, presque naturellement, celle du 4 âge caractérisée par l'immobilité et l'incapacité liées aux problèmes de santé et à la dépendance. Au final, si le $3^{\text {ème }}$ âge correspond à la période où cessent les activités professionnelles, le $4^{\text {ème }}$ âge désigne celle au cours de laquelle les handicaps apparaissent et où la plupart des activités deviennent difficiles ${ }^{4}$. Compte tenu de la fluctuation de l'âge de départ à la retraite et de l'amélioration des conditions de vieillissement, les $3^{\text {ème }}$ et $4^{\text {eme }}$ âges s'imposent comme des notions qualitatives qui s'efforcent de préciser la capacité sociale des individus à agir (en activité professionnelle ou pas, empêchés ou pas...). Les sociologues ont d'ailleurs assimilé les liens qui unissent l'émergence de la catégorie du $4{ }^{\text {ème }}$ âge à l'apparition des notions de dépendance, de perte d'autonomie ${ }^{5}$ et aux politiques sociales associées (Caradec, 2008).

Outre la manière dont les formes de solidarité intergénérationnelle sont actuellement repensées pour assurer la continuation du système de retraite par répartition, les plus de 65 ans désignent, à de rares exceptions près ${ }^{6}$, la population des retraités en France. Mais opter pour une catégorie d'âge plutôt que pour une catégorie sociale comme celles des seniors ou du $3^{\text {ème }}$ âge, c'est à la fois dépasser l'assignation socioprofessionnelle des individus, et homogénéiser une catégorie loin de l'être.

Si l'on s'en tient à la question des pratiques culturelles, il y a nécessité, comme nous le rappelle Philippe Chantepie ${ }^{7}$, d'imaginer de nouvelles offres en matière de pratiques culturelles pour des retraités qui représentent une population qui se développe sans cesse, avec une espérance de vie qui s'allonge, toujours en meilleure santé, et de plus en plus active et participative ${ }^{8}$. Encore une fois, ces tendances ne doivent pas faire oublier les nombreuses inégalités qui caractérisent la vieillesse qui doit faire face aux problèmes de santé, notamment dans les catégories les plus précaires qui sont aussi celles qui ont eu des conditions de travail difficiles.

Toutes ces remarques nous permettent de préciser que les plus de 65 ans dont nous parlons ici désignent la catégorie plutôt privilégiée de la population vieillissante que la notion de seniors désigneraient mieux si nous n'avions pas voulu davantage porter l'attention sur les inactifs de ce groupe. L'utilisation d'une tranche d'âge relève ici davantage d'une commodité à l'égard de nos données que d'une volonté de parler de manière exhaustive des plus de 65 ans, dont les fractions les plus âgées ne sont que très peu représentées parmi les festivaliers.

\subsection{Une palette singulière de goûts}

\footnotetext{
${ }^{3}$ P. Laroque, Rapport de la Commission d'Etude des problèmes de la Vieillesse, 1963

${ }^{4}$ Une étude de l'INSEE datant de 1996 montre que 28\% des personnes de plus de 80 ans vivant chez elles manquent d'autonomie (contre $11 \%$ des $60-80$ ans) et $72 \%$ ont besoin d'aide pour un certain nombre de tâches domestiques (ménages, courses, repas...).

M.G. David et Starzec C.. « Aisance à 60 ans, Dépendance et isolement à 80 ans », Insee Première, 1996.

${ }^{5}$ M. Ségalen, Sociologie de la famille, Paris, Armand Colin, 2010.

${ }^{6}$ Selon l'INSEE, en moyenne en 2009, 173000 personnes de 65 ans et plus ont un emploi, soit 1,7\% de l'ensemble des individus âgés de 65 ans et plus. Source : Insee, enquêtes Emploi du 1er au 4ème trimestre 2009).

${ }^{7}$ Philippe Chantepie est chef du Département des études, de la prospective et des statistiques (DEPS).

${ }^{8}$ Les retraités deviennent progressivement un public qui électoralement sera de plus en plus dominant comme le précise Monique Boutrand (dans un rapport intitulé "Sénior et cités » et réalisé en 2009 pour le Conseil Economique, Social et Environnemental) les plus de 60 ans représentent aujourd'hui plus de $25 \%$ du corps électoral, en 2020, il avoisinera les $40 \%$.
} 
La variété de notre échantillon en termes de spectacles et de genre musicaux, de même que les préférences musicales exprimées par les festivaliers permettent d'explorer un nouveau domaine d'interrogation, celui des goûts. Pour cela, nous allons comparer les préférences déclarées des festivaliers de plus de 65 ans à ceux qui ont moins de 40 ans. De la sorte, nous pourrons tenter d'identifier, si elle existe, la spécificité d'un profil de goûts propres aux plus de 65 ans.

\section{(1) Profil moyen et méthode de calcul}

Le profil moyen des goûts des festivaliers a été obtenu à partir des genres musicaux suivants : lyrique ; musique sacrée ; musique médiévale ; baroque ; classique ; musique contemporaine ; jazz, blues, musiques improvisées; musiques actuelles, rock, rap, hip hop ; musiques du monde ; chanson, variétés ${ }^{9}$. Chaque genre a ensuite été noté en fonction des quatre niveaux de réponse proposés dans le questionnaire (beaucoup ; assez ; un peu ; pas du tout). Nous avons enfin confronté ces différentes notations aux variables suivantes: âge, provenance géographique, catégorie socioprofessionnelle, sexe, pratique instrumentale, connaissance de la programmation et degré de fidélité au festival. Il nous est donc possible d'isoler cette population des plus de 65 ans - qui représentent environ $20 \%$ de notre échantillon (soit plus de 4000 individus) -, d'analyser sa diversité interne et de la confronter aussi au groupe des moins de 40 ans (environ 6000 individus).

Figure 2. Comparaison des goûts musicaux des plus de 65 ans et des moins de 40 ans

\footnotetext{
${ }^{9}$ Le détail accordé aux musiques savantes, comparé aux catégories plus composites des autres répertoires musicaux, pourra surprendre, à raison, le lecteur soucieux de respecter la spécificité des différents genres. Ces regroupements résultent d'un choix imposé par la spécificité de l'échantillon initial de notre étude, davantage orienté vers des festivals de musiques savantes. Dans un souci de comparaison, nous n'avons pas choisi de changer cette classification lorsque nous avons pu étendre notre enquête aux festivals de musiques actuelles, de variétés, de chanson et de musiques du monde.
} 


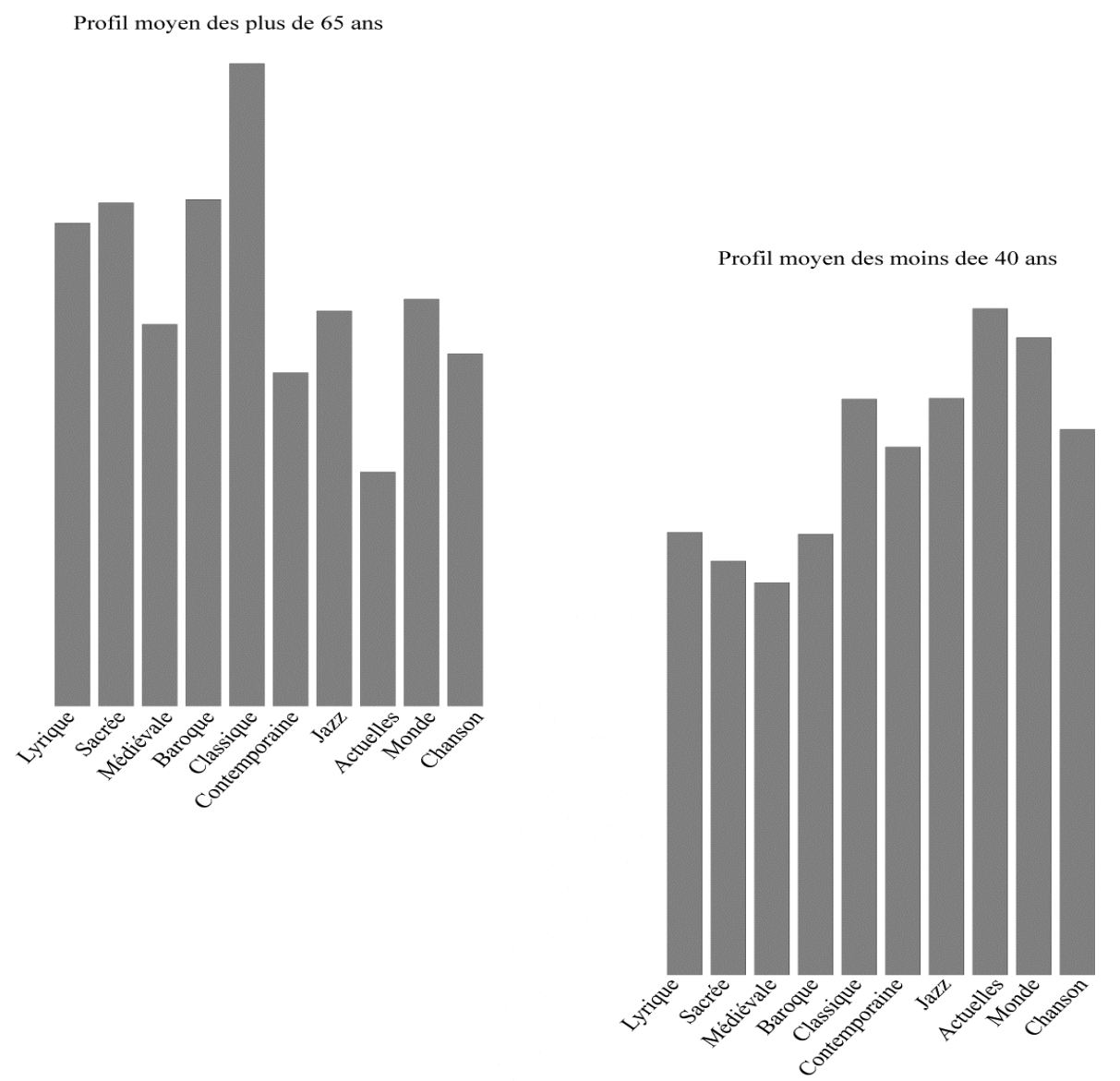

Source : Enquête sur les publics des festivals de musique et de danse 2008, $(\mathrm{n}=23$ 344).

La confrontation des deux profils moyens montre que certains traits opposent les palettes de préférences entre personnes âgées et jeunes adultes. D'un côté, les festivaliers de plus de 65 ans, dont la moyenne d'âge est de 70 ans, expriment des préférences du côté des musiques savantes, avec une nette domination de la musique classique, et une certaine ouverture, du côté «moderne » vers le jazz. Le genre le plus rejeté est ici celui des musiques actuelles, du rock au rap et hip-hop. Ces plus de 65 ans semblent ainsi confirmer le rôle du facteur générationnel dans l'approche des goûts, sans pour autant que l'on puisse y voir une prédiction: rien ne permet en effet d'en tirer une conclusion sur la «classicisation» (Donnat 2009) des goûts au fil de la vie, et singulièrement du vieillissement. L'orientation vers les musiques savantes, nous le verrons, résulte à la fois d'héritages culturels propres à une certaine appartenance sociale, à une période et à une génération. Comme l'indique Olivier Donnat, rien ne dit pour autant - il semble même pencher pour le contraire (Donnat 2009: 132-136) - que la génération actuelle des quadragénaires, qui est plutôt adepte de musiques actuelles, se tournera vers la musique classique à l'heure de sa maturité.

Il reste que l'opposition des palettes est évidente entre les populations les plus jeunes et les plus âgées. Les premières privilégient nettement les répertoires les plus actuels, qui vont des formes les plus « classicisées »-comme le jazz -, à des genres mixtes - comme les musiques du monde ou la chanson - en passant par des formes proches de la musique savante - comme le classique - ou, au contraire, des musiques amplifiées proches du blues ou du rock. Les secondes ont des préférences plus marquées, elles-mêmes diversifiées, en faveur des répertoires savants. 


\section{(2) Les moins de 40 ans : entre éclectisme et repli sur les musiques actuelles}

Chez les moins de 40 ans, on remarque que les profils sont assez distincts en fonction de certaines caractéristiques. Ainsi, les profils qui associent un goût avéré pour les musiques actuelles tout en réservant un très bon accueil à des formes plus savantes (comme le lyrique ou le classique par exemple). On remarque alors que ceux des moins de 40 qui ont le plus tendance à opérer cette ouverture vers le classique sont en même temps les plus âgés de leur groupe (31 ans contre 27 ans en moyenne), qu'ils appartiennent davantage aux classes supérieures (69\% contre 53\% en moyenne dans cette catégorie) et qu'ils pratiquent davantage d'un instrument.

À l'opposé, les profils les plus exclusivement actuels, chez qui les musiques savantes n'ont pas voix au chapitre, sont en même temps plus populaires (39\% de classes populaires contre $20,5 \%$ en moyenne dans cette tranche d'âge), moins portés vers la pratique amateur d'un instrument (48\%, contre $60 \%$ en moyenne), mais ne sont pas pour autant plus jeunes. Chez les moins de 40 ans, il semble donc y avoir une relation assez claire entre génération, appartenance sociale et pratique instrumentale, ces trois variables étant celles qui varient le plus fortement d'un profil à l'autre. En est-il de même pour les générations les plus âgées?

\section{(3) Les plus de 65 ans : entre éclectisme et repli sur la musique savante}

$\mathrm{Au}$ sein de cette population aux préférences essentiellement tournées vers les musiques savantes, trois autres profils se dégagent.

Figure 3. Les trois profils gustatifs des plus de 65 ans

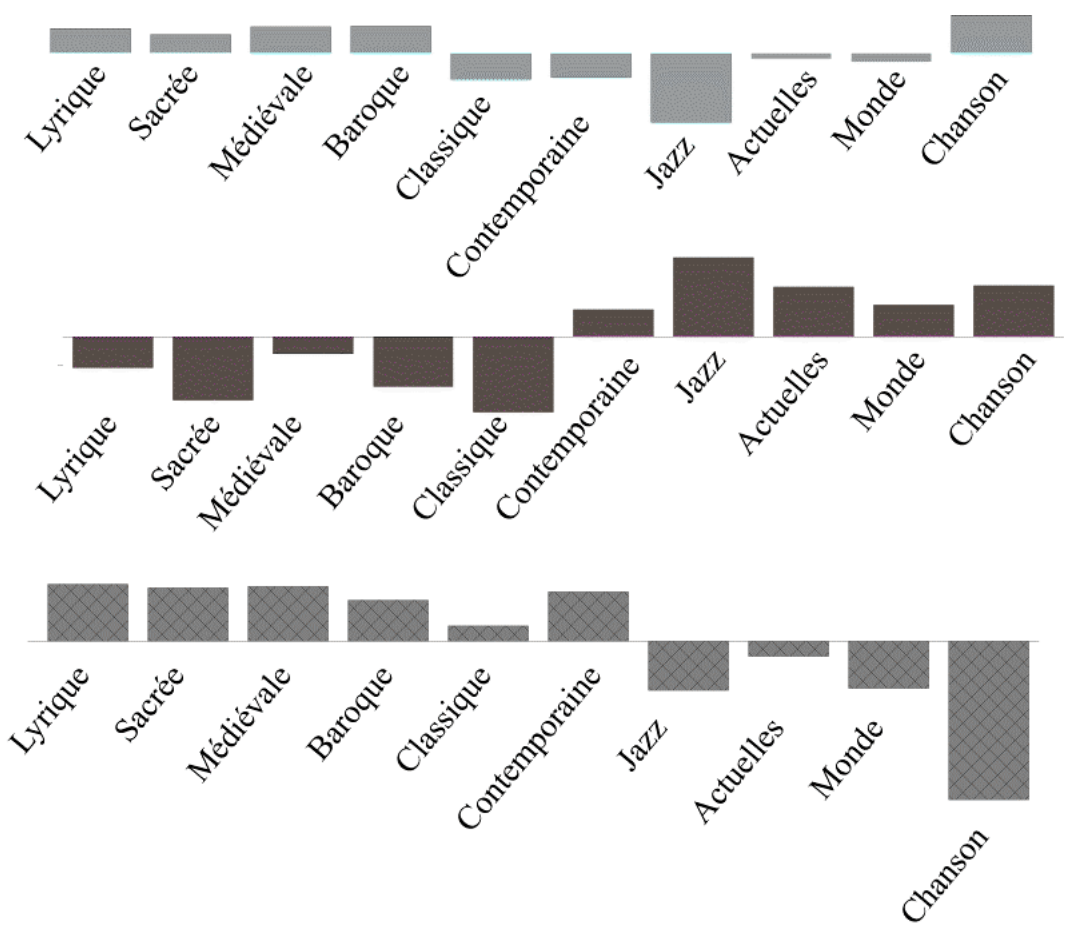

Source : Enquête sur les publics des festivals de musique et de danse 2008, (n=23 344). 
Le premier de ces trois profils valorise un peu plus encore les répertoires savants - à l'exception notable du classique et de la musique contemporaine - tout affirmant un goût particulièrement prononcé pour la chanson. On trouve ici des spectateurs plutôt habitués du festival où ils ont été interrogés $(80 \%$ ont déjà assisté à au moins une édition antérieure, contre $61 \%$ en moyenne) et davantage issus des classes supérieures $(66 \%$ contre $58 \%$ en moyenne).

Plus éclectique, le second profil se distingue par une survalorisation de l'ensemble des répertoires modernes et populaires, et une nette prédilection pour le jazz. Sociologiquement distincts du profil précédent, les festivaliers rassemblés ici sont davantage issus des classes moyennes et populaires. Ils sont moins fidèles aussi (75\%).

Le troisième profil désigne, enfin, l'archétype du festivalier féru de musiques savantes, à l'exclusion de tout autre genre. A l'opposé du premier, ce groupe se caractérise, en outre, par un rejet massif de la chanson. Pourtant, sociologiquement, leur profil est assez comparable au premier : PCS élevée, fidélité maximale $(81 \%)$ et âge légèrement plus élevé que la moyenne de cette catégorie ( 71 ans)

Quelle conclusion tirer de ces profils ? Tout d'abord un constat : il n'y a pas, chez les plus de 65 ans, une communauté de goûts homogènes mais bien des palettes de goût assez variés. Si l'âge oriente les goûts des festivaliers, il ouvre sur des chemins distincts en fonction de l'appartenance sociale. Il n'y a donc pas lieu de "fétichiser» l'âge comme variable permettant de tout expliquer.

Qu'en est-il du lien établi, au cours de la dernière décennie, entre l'éclectisme et l'appartenance sociale (Peterson 1992, Donnat 1994) ? Celui-ci indique que ce n'est pas tant le monopole du goût pour les esthétiques les plus savantes qui marquerait les catégories sociales supérieures, que l'élargissement de leur palette gustative, autrement nommé « omnivorisme ». En réalité, nous notons ici que, contrairement à une idée souvent répandue, les profils qui sont les plus prompts à accueillir les genres les plus divers ne sont pas nécessairement les plus favorisés socialement. L'éclectisme ou l'omnivorisme (Peterson 2004) ne semblent pas être l'apanage des catégories les plus aisées au sein de cette population de plus de 65 ans. Autrement dit, il n'y a pas de continuum entre appartenance sociale élevée et ouverture plus large des palettes de goûts.

Il y a donc là une différence entre nos deux groupes. Si, pour les plus jeunes, l'ouverture de la palette des goûts est en général le fait des catégories sociales supérieures, c'est l'inverse qui se produit chez les personnes âgées où les festivaliers ayant la palette de goûts la plus ouverte sont ceux issus des classes moyennes et populaires. Cela indique au moins la résistance d'une certaine tendance légitimiste qui fait, chez les jeunes, de l'ouverture aux musiques savantes le produit d'un certain héritage social et culturel. Mais cela montre aussi que l'ouverture symétrique (depuis les musiques savantes vers des répertoires plus populaires) porte également la marque des appartenances sociales. Au final, la variable de l'âge permet de montrer que le lien entre appartenance sociale et répertoires de goûts ne relève pas de l'évidence, et il se pourrait bien que pour les futures générations de plus de 65 ans, l'avancée dans l'âge soit également l'occasion d'expérimenter de nouveaux répertoires de goûts. Plus prononcé chez les classes moyennes et populaires, ce comportement pourrait bien témoigner d'un des effets positifs des politiques de démocratisation culturelle. 
De fait, cette hétérogénéité des répertoires de goûts à l'intérieur des différentes classes d'âges, nous incite à reconsidérer la variable de l'âge, non plus comme un critère fixe (plus de 65 ans), mais comme une variable relative.

\section{L’ÂGE ET SES EFFETS RELATIFS}

Nous avons raisonné jusqu'ici sur la catégorie fixe des plus de 65 ans, sans nous interroger sur les effets de l'âge en termes relatifs. Nous souhaitons les mesurer maintenant à partir d'une autre mesure : celle des $10 \%$ de festivaliers les plus âgés de chaque festival, confrontée à la fraction de $10 \%$ la plus jeune. Nous allons l'observer à partir d'un échantillon de festivals dont les caractéristiques et l'âge moyen sont très différents: deux festivals de musiques actuelles (Chapiteuf et Transes Cévenoles); deux festivals de musique classique (Saintes et Montpellier Radio-France) ; deux festivals de jazz (Junas et Sète) et deux festivals de danse (Montpellier danse et La Biennale de Lyon). Pour ces festivals, nous allons repérer les éventuels écarts qui existent entre ces deux fractions de publics (les 10\% plus âgés, et les 10\% plus jeunes) à propos de leurs profils moyen de goût, de leurs pratiques culturelles, de leur origine géographique et de leur degré de fidélité au festival.

En observant maintenant les écarts qui existent entre les fractions les plus jeunes et les plus âgées des publics de ces festivals, nous cherchons à identifier le rôle de l'âge dans l'orientation des goûts musicaux, l'intensité des pratiques culturelles, la mobilité et la fidélité des publics. Son impact est différencié en fonction de plusieurs paramètres, comme nous allons le voir au travers d'une sélection de résultats les plus significatifs.

\subsection{Les goûts}

Pour les goûts, nous avons calculé la note moyenne accordée, à chaque genre de musique, par les plus jeunes et par les plus âgés de chaque festival. L'écart entre les deux est variable selon les genres. Nous mesurons ensuite les écarts en valeur absolue, pour évaluer l'ampleur des différences de goût entre les deux groupes d'âge. On notera ainsi qu'il y a bien moins d'écart entre ces deux "générations » en musiques actuelles qu'en classique ou, surtout, en danse ! Nous faisons, enfin, le lien entre les moyennes d'âge des festivals, les intervalles d'âge entre groupes, et les différences de goût, de manière à observer l'influence de l'âge relatif sur les goûts.

Figure 4. Les goûts à travers les âges 


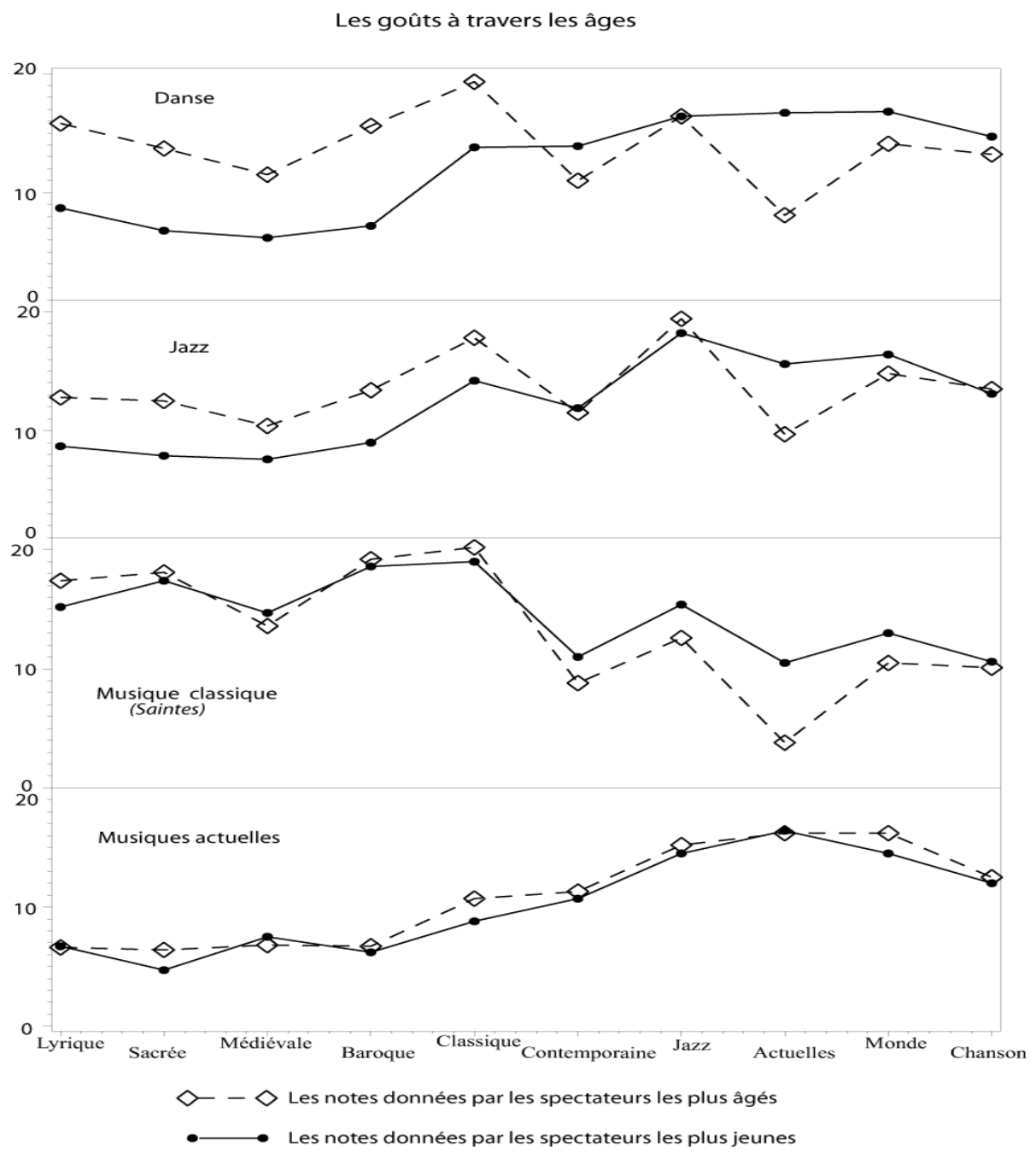

Source : Enquête sur les publics des festivals de musique et de danse 2008, (n=23 344).

Cette série de graphiques et le tableau 5 nous conduisent à plusieurs enseignements confirmés par l'examen d'autres publics de festivals, que nous ne présentons pas ici. Le premier est la confirmation de ce que nous indiquions dans la partie précédente : il y a une influence de l'âge sur l'orientation des goûts. Les plus anciens «relatifs" (c'est-à-dire les 10\% de festivaliers les plus âgés de chaque festival) sont toujours plus ouverts aux musiques savantes que les autres, tandis que, réciproquement, les plus jeunes le sont davantage à l'égard des musiques actuelles.

Tableau 2. Les différences de goût, l'âge moyen et les écarts entre fractions d'âge ${ }^{10}$

\begin{tabular}{|l|r|r|r|}
\hline Festivals & Âge moyen & $\begin{array}{r}\text { Écart entre } \\
\text { moyennes }\end{array}$ & $\begin{array}{r}\text { Écarts } \\
\text { cumulés } \\
\text { entre } \\
\text { groupes }\end{array}$ \\
\hline Musiques actuelles & 30 & 20 & 9 \\
\hline Danse Lyon & 42 & 32 & 37 \\
\hline
\end{tabular}

\footnotetext{
${ }^{10}$ La première colonne du tableau indique l'âge moyen des festivaliers, la seconde indique l'écart d'âge entre le groupe des plus jeunes et celui des moins jeunes, enfin la dernière colonne indique la différence de goûts entre ces deux groupes (en valeur absolue).
} 


\begin{tabular}{|l|l|l|l|}
\hline Danse Montpellier & 46 & 33 & 49 \\
\hline Jazz & 50 & 23 & 29 \\
\hline Classique Montpellier & 55 & 21 & 26 \\
\hline Classique Saintes & 60 & 17 & 20 \\
\hline
\end{tabular}

Source : Enquête sur les publics des festivals de musique et de danse 2008, ( $\mathrm{n}=23$ 344).

Mais ce résultat, assez attendu, est bien moins intéressant que l'examen des écarts entre goûts, que fournit la somme des valeurs absolues (colonne de droite). Et là nous observons que cet écart est très faible pour les musiques actuelles, un peu plus fort pour les musiques savantes, et encore plus fort pour le jazz et la danse, où il est à son maximum. Comment l'expliquer ? D'une part, s'agissant des musiques actuelles, nous sommes face à ce que Hervé Glévarec et Michel Pinet ont identifié comme l'émergence d'une préférence exclusive pour les genres populaires chez les moins de 40 ans, et d'un éclectisme dominé par les genres populaires chez les plus de 40 ans (Glévarec et Pinet 2009, p.614). Dans l'univers des musiques actuelles, l'écart entre jeunes et moins jeunes est très faible, et l'ouverture vers les musiques savantes reste sélective et modérée.

Par ailleurs, l'écart entre tranches d'âge reste également limité chez les festivaliers de musiques savantes. Ainsi, dans le cas de Saintes, l'ouverture - due à l'âge - vers les musiques actuelles et le jazz reste également limitée chez les $10 \%$ de spectateurs les plus jeunes, mais dont l'âge peut atteindre tout de même 52 ans. Autrement dit, tel que nous l'observons dans les deux sens, la modération de l'ouverture aux goûts savants, d'un côté, et populaires, de l'autre, est le fait de groupes dont les âges moyens sont les plus rapprochés : 20 ans pour les musiques actuelles, et 17 ans à Saintes.

En revanche, dès que l'écart entre ces groupes jeunes et moins jeunes se creuse, les différences de goût ont tendance à croître. En jazz, avec un écart de 23 ans entre l'âge maximum des plus jeunes et l'âge minimum des plus âgés, la différence de goût atteint un score de 29. En danse, à Montpellier, avec un écart de 33 ans entre les mêmes populations, on atteint un score record de 48,9.

L'influence de l'âge est donc une donnée importante, y compris en termes relatifs. Plus les écarts entre tranches d'âge s'élèvent et plus les différences de goût s'accroissent. En somme, les populations les plus âgées tendent, au-delà de la diversité réelle des univers gustatifs que nous avons mis en évidence dans la partie précédente, à être plus nettement orientées vers les registres savants et "légitimes". Mais cette tendance décroît notamment avec le type de programmation fréquentée. Ainsi, les spectateurs les plus âgés de festivals de musique « populaire » ont certes une orientation plus prononcée en faveur du classique, mais l'ampleur de l'écart avec les plus jeunes y est moins forte que dans les publics de musiques savantes.

Qu'en est-il cependant des pratiques culturelles ? Montrent-elles des tendances similaires?

\subsection{Les pratiques culturelles}

Après avoir vu que les plus âgés avaient tendance à se tourner vers les registres savants, il est intéressant de s'arrêter sur leurs pratiques culturelles : ont-ils des pratiques culturelles plus légitimes et plus intenses que les plus jeunes? 
Tableau 3 : L'âge et les pratiques culturelles ${ }^{11}$

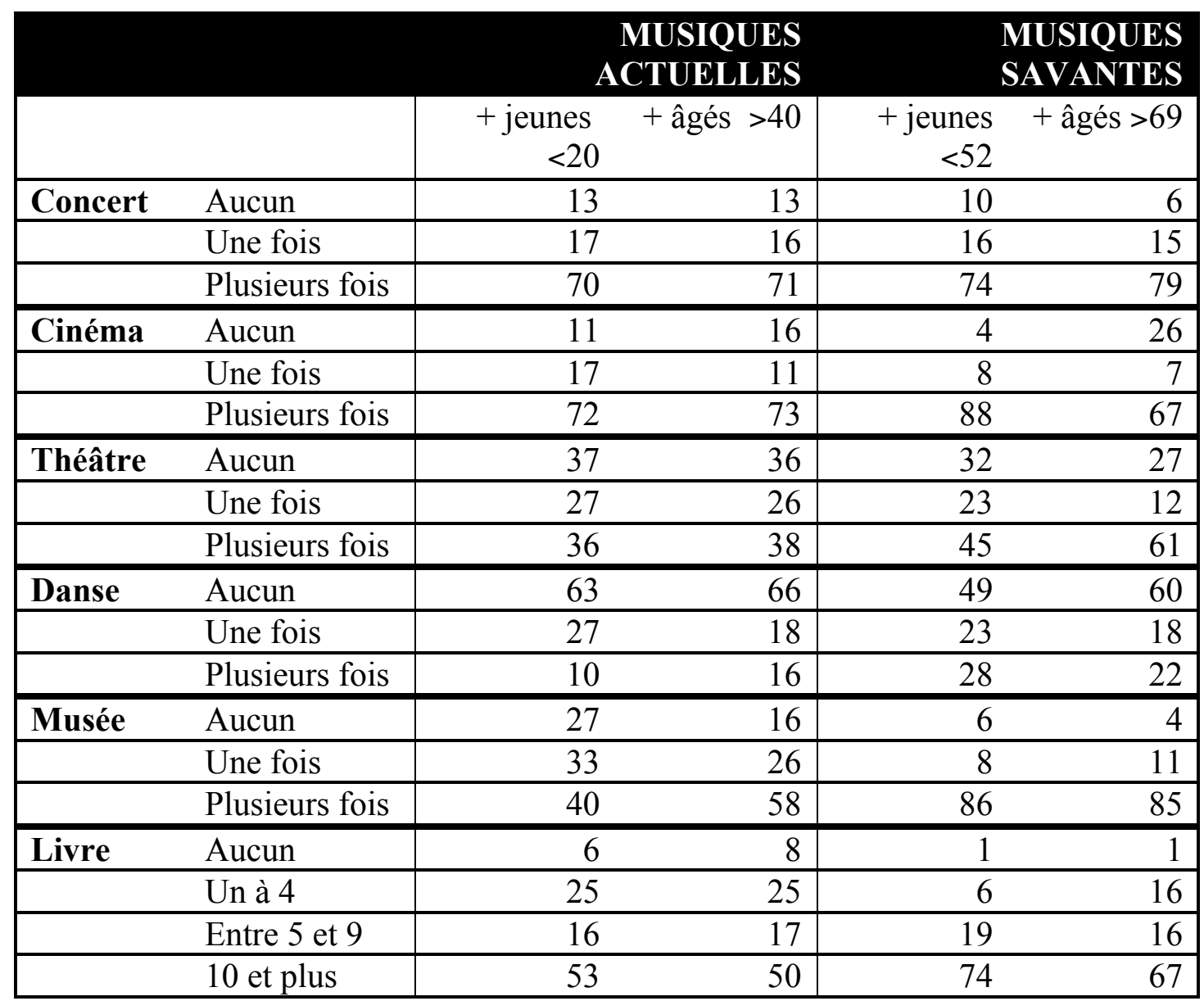

Source : Enquête sur les publics des festivals de musique et de danse 2008, $(\mathrm{n}=23$ 344).

Ce tableau est très significatif des tendances que nous observons sur l'échantillon plus large des publics des festivals. Partant d'une assez grande correspondance entre la pratique festivalière et les pratiques culturelles tout au long de l'année, nous pouvons cependant distinguer ce qui relève en propre des pratiques culturelles des personnes les plus âgées. Ici encore, c'est l'âge relatif qui nous intéresse. Ce que montre ce tableau est tout d'abord une confirmation des données sur les pratiques culturelles des français, au moins sur deux points.

Tout d'abord, les festivaliers âgés ont une palette de pratiques distincte de celle des jeunes. Chez les premiers, on peut observer l'influence, supérieure à ce qu'elle est chez les jeunes, de pratiques « classiques » comme la fréquentation de musées et d'expositions, de livres et, dans une moindre mesure, de danse et de théâtre. Ensuite, les festivaliers âgés ne sont qu'exceptionnellement des spectateurs ponctuels : seuls $6 \%$ des plus âgés de notre échantillon n'avaient pas fréquenté le moindre concert avant de répondre à nos questions à l'occasion d'un festival. Le fait que cette pratique croisse encore avec l'âge - les plus jeunes de cet échantillon, à moins de 52 ans, sont $10 \%$ dans ce cas - est un clair indicateur du dynamisme des pratiques culturelles des seniors.

\footnotetext{
${ }^{11}$ Ce tableau représente la part des festivaliers selon les pratiques culturelles, les tranches d'âge et le type de musique. Par exemple : dans les musiques actuelles, 36\% des festivaliers les plus jeunes sont allés plusieurs fois au théâtre au cours de l'année contre $38 \%$ des plus âgés.
} 
Mais celui-ci n'est pas sans limite. Comme on peut le constater, les festivaliers les plus âgés, s'ils ont une pratique croissante de concerts ou de théâtre, voient décliner la plupart des autres pratiques culturelles : danse, cinéma, lecture notamment.

\subsection{Un public fidèle ?}

Concernant le renouvellement des publics, l'hypothèse courante serait que le public plus âgé est systématiquement plus fidèle que le plus jeune. Cette loi se vérifie clairement. Il y a toujours plus de nouveaux festivaliers (qui n'étaient jusque là jamais venus à cette manifestation) que d'anciens. Ce qui est intéressant ici, c'est plutôt de voir que, à l'instar de ce que nous avons indiqué à propos des goûts, c'est aux extrêmes que les écarts entre jeunes et plus âgés sont les plus faibles. Rappelons que le taux moyen de renouvellement des publics, dans notre étude de 2010, est de 39\% (Négrier, Djakouane et Jourda 2010). Le tableau $n^{\circ} 8$ compare la part des nouveaux spectateurs dans les tranches d'âge les plus jeunes et les plus âgées des festivals. Il nous incite à penser, s'agissant d'événements qui ont tous plus de sept ans d'ancienneté, que le fort renouvellement des publics va toujours de pair avec le rajeunissement des festivals, même pour ceux qui ont des publics à moyenne d'âge élevée. Cette influence de l'âge est renforcée par le constat suivant : c'est encore une fois pour les festivals de danse, où l'écart d'âge entre les $10 \%$ plus jeunes et $10 \%$ plus âgés des publics est le plus fort, que le différentiel de renouvellement des publics est également le plus large.

Tableau 4. L'âge des nouveaux festivaliers

\begin{tabular}{|lrrr|}
\hline Programmation/Festivals & + jeunes & + âgés & Écart \\
\hline Musiques actuelles & $59 \%$ & $55 \%$ & -4 \\
\hline Jazz & $73 \%$ & $36 \%$ & -37 \\
\hline Danse Lyon & $80 \%$ & $34 \%$ & -46 \\
\hline Danse Montpellier & $61 \%$ & $20 \%$ & -41 \\
\hline Montpellier Radio-France & $42 \%$ & $15 \%$ & -27 \\
\hline Saintes & $31 \%$ & $12 \%$ & -19 \\
\hline
\end{tabular}

Source : Enquête sur les publics des festivals de musique et de danse 2008, ( $\mathrm{n}=23$ 344).

\section{CONCLUSION}

Alors qu'en 2008, 45\% des plus de 65 ans n'avaient pas fréquenté d'équipements culturels au cours de l'année, ces derniers représentent malgré tout $20 \%$ du public des festivals de musique et de danse. Sans grande surprise, ces festivaliers se trouvent principalement dans les festivals où dominent les répertoires de musique savante. Certes, il existe ce que l'on pourrait nommer des festivals de « seniors » où la part des plus de 65 ans et la moyenne d'âge sont relativement importantes. Pour autant, les jeunes n'en sont pas moins exclus et ne renient pas la musique savante.

Confrontée à l'étude des répertoires de goûts, la mixité générationnelle autour des musiques savantes nous a ainsi permis de montrer que, tout comme il n'existe pas de festivals de « seniors ", il n'existe pas une palette de goûts musicaux propre à chaque génération. Même si la pluralité des univers gustatifs connaît ses limites, chez les plus jeunes comme chez les plus âgés. C'est ainsi plusieurs profils de goûts hétérogènes qui cohabitent au sein de chaque génération.

L'analyse du poids de l'âge relatif nous a ainsi permis de mettre en évidence l'importance de cette variable dans l'orientation des goûts musicaux, l'intensité des pratiques culturelles, et la fidélité des publics. Les festivaliers les plus âgés ont une palette de pratiques et de goûts bien 
distincte de celle des jeunes. Ces différences de goûts et de pratiques semblent être croissantes avec l'écart d'âge entre les populations. Plus les écarts d'âge entre les plus jeunes et les plus âgés des festivaliers s'élèvent et plus les différences de goût s'accroissent et plus les personnes âgées sont fidèles. Cette analyse quantitative des festivaliers seniors nous a permis de dresser divers portraits et de comparer diverses catégories de population. Elle pallie le manque de données concernant les pratiques culturelles des personnes âgées mais pour autant, elle ne nous dit rien sur l'évolution des goûts au cours du cycle de vie. 


\section{Références}

ARINO, A. (2010). Prácticas culturales en España. Desde los años sesenta hasta la actualidad, Barcelona : Ariel

ATTIAS-DONFUT C., SEGALEN M. (1997). Grands Parents. La famille à travers les générations, Paris, Odile Jacob

BOUTRAND M (2009). Seniors et cité, Rapport du COR pour le Conseil Economique, Social et Environnemental

CARADEC V., (2005). Sociologie de la vieillesse et du vieillissement, Paris, Armand Colin

CARADEC V., (2003). «Comportements culturels de la population âgée », in EMPAN, n52, 54-61

DELBES C., GAYMU J. (1995). «Le repli des anciens sur les loisirs domestiques. Effet d'âge ou de génération », Population, n³, 689-720

DONNAT O., LEVY F. (2007). "Approche générationnelle des pratiques culturelles et médiatiques », Culture et prospective, $\mathrm{n}^{\circ}$ 2007-3

DONNAT, O. (1994). Les Français face à la culture : de l'exclusion à l'éclectisme, Paris, La Découverte

DONNAT, O.(2001). « Sociologie des pratiques culturelles », in P. Poirrier (dir.), Politiques et pratiques de la culture, Paris, La Documentation Française, 2010, 193-201

DONNAT, O. (2009). Les pratiques culturelles des français à l'ère numérique, Paris, La Documentation Française

GLEVAREC H., PINET M. (2009). «La « tablature » des goûts musicaux : un modèle de structuration des préférences et des jugements », Revue Française de Sociologie, 50-3, 599640

GUERIN, S. (2009). « Introduction au cahier : La société des seniors, jeunesse d'un défi », Management \& Avenir 10/2009, n 30, 75-77

NEGRIER E., DJAKOUANE A. et JOURDA M. (dir.) (2010), Les publics des festivals, Paris : Michel de Maule

PAILlAT P. (dir.) (1993). Les Pratiques culturelles des personnes âgées, Paris, La Documentation française

PETERSON R. A.(1992). «Understanding Audience Segmentation : from Elite and Mass to Omnivore and Univore », Poetics, 21-4, 243-258

PETERSON R. A. (2004). «Le passage à des goûts omnivores : notions, faits et perspectives », Sociologie et Sociétés, 36, 1, 145-164 